\title{
El historiador y la utopía. Reflexiones inspiradas en una clase magistral
}

\section{The historian and utopia. Reflections inspired by a master class}

Rafael Vega Centeno Sara Lafosse ${ }^{1}$

Pontificia Universidad Católica del Perú

\section{Resumen:}

A Jeffrey Klaiber se le reconoce por su obra prolífica, caracterizada por una metódica recuperación y presentación de información. Su cuidado por la objetividad en el juicio histórico no significó, sin embargo, una ausencia de involucramiento o de toma de posición frente al devenir histórico. El texto Globalización y evangelización en los siglos XVI y XXI. Ideología frente a utopia, que es una lección inaugural del año 2005 en la Universidad Antonio Ruiz de Montoya, pone en evidencia su compromiso y apuesta por la utopía, entendida como la acción humana orientada a materializar sueños y anhelos de una sociedad mejor.

Palabras clave: utopía, ideología, evangelización, misiones, siglo XVI

1 Pontificia Universidad Católica del Perú, Departamento de Humanidades. Código ORCID: 0000-0001-8519-3590. Contacto: rvegacen@ gmail.com 


\section{Abstract:}

Jeffrey Klaiber is recognized for his prolific work, characterized by a methodical retrieval and presentation of information. His care for objectivity in the historical judgment did not mean, however, an absence of involvement or of taking a position in the historical process. The text Globalization and Evangelization in the XVI and XXI centuries. Ideology versus utopia, which is an inaugural lecture of the year 2005 at the Antonio Ruiz de Montoya University, puts in evidence his commitment and bet for utopia, understood as the human action aimed at materializing dreams and longings for a better society.

Keywords: utopia, ideology, evangelization, missions, $16^{\text {th }}$ century

Jeffrey Klaiber es reconocido como un historiador prolífico con un notable talento para la recolección de información y su presentación prolija, que permite al lector una introducción rápida y amigable a los temas tratados.

En distintos foros (y en este mismo volumen) se destaca también el cuidado que tenía Klaiber para presentar el hecho histórico de forma objetiva sin introducir juicios de valor personal. A través de sus escritos, se evidencia que él estaba lejos del perfil de "científico social militante" común entre

288 historiadores, sociólogos y otros científicos sociales de la segunda mitad del siglo XX.

Estas características de la obra de Klaiber pueden llevar a cuestionamientos sobre su grado de concernimiento o, incluso, su compromiso con el devenir histórico. Llevado a un extremo, se podría crear la imagen de un historiador ajeno a los desafíos de la humanidad, escasamente involucrado con las interpelaciones que la historia nos presenta. 
Con toda seguridad, los testimonios de quienes conocieron a Klaiber pueden desvirtuar esos juicios. Pero más allá de la experiencia personal, considero que el compromiso de Klaiber como historiador y docente quedó evidenciado en el texto Globalización y evangelización en los siglos XVI y XXI. Ideología frente a utopia ${ }^{2}$. Se trata de una transcripción de la lección inaugural que Klaiber ofreció como parte de la apertura del año académico 2005 en la Universidad Antonio Ruiz de Montoya, en la que fue profesor desde su fundación y llegó a ser decano de la Facultad de Educación.

El texto en mención no proporciona necesariamente nueva información en relación con trabajos anteriores, sino que aborda un tema estudiado por Klaiber a lo largo de su obra, como es la historia de la Iglesia en América Latina. Testimonio de esa dedicación fue su participación en el volumen sobre Ecuador, Perú y Bolivia, de la colección Historia General de la Iglesia en América Latina, elaborada por la Comisión de Estudios de Historia de la Iglesia en América Latina (CEHILA) o su estudio sobre el accionar político de la Iglesia a fines de la colonia (Klaiber, 2013) y los avatares de la iglesia a lo largo del siglo XX latinoamericano entre dictaduras y democracias (Klaiber, 1997). En esa misma línea de indagaciones, se incorpora un estudio general de la historia de la Compañía de Jesús en Latinoamérica (Klaiber, 2007). La singularidad del texto de la lección inaugural no está en la presentación de nueva información para investigadores o especialistas, sino en la lectura que Klaiber da a la información conocida en el contexto de una exposición para un claustro universitario.

2 El texto fue publicado en el libro La identidad ignaciana de la Universidad Antonio Ruiz de Montoya, editado por V. Santuc (ed.). Lima: Universidad Antonio Ruiz de Montoya, 2006. 
En el título de la disertación, Klaiber (2006) plantea una comparación entre las coyunturas de los siglos XVI y XXI por ser tiempos de descubrimientos, de nuevos experimentos y de un mundo conectado. Sobre la base de estos elementos comunes, nos invita a revisar la historia de la evangelización en América Latina a través del tamiz de la reciente globalización. Lo más importante es, sin embargo, la inclusión de un esquema cíclico en la linealidad del devenir histórico, como marco para entender el comportamiento de la Iglesia en América Latina. Así, la historia de la Iglesia consistiría en un constante ir y venir entre la utopía y la ideología (p. 147).

Para ilustrar esos escenarios, Klaiber se apoya en los conceptos desarrollados por Karl Manheim (1973), quien definía la utopía como una coyuntura en la que la acción de los seres humanos se orienta a materializar sueños y anhelos de una sociedad proyectada, significativamente mejor que la existente. En contraste, la ideología es la coyuntura, cuya práctica se orienta mayoritariamente a "congelar" o "domesticar" dichos sueños para evitar transformaciones sociales de resultados no previsibles.

La historia se nos presenta, así, como una alternancia de ambos tipos de contextos. No se trata, sin embargo, de ciclos monolíticos, sino que es posible encontrar contextos mixtos con posiciones utópicas e ideológicas que se confrontan o en los que, siendo una de ellas dominante, no inhibe la existencia de otras. Klaiber toma claramente posición por la perspectiva utópica y reconoce, por otro lado (y quizás por eso mismo), ante el claustro que lo escuchaba, que vivimos en un contexto con posiciones ideológicas dominantes (2006, p. 157). Revisemos en detalle el desarrollo de la disertación.

Esta comienza poniendo en relieve los inicios de la evangelización en América, en la que se manifestaron declaraciones y prácticas claramente utópicas, de mucha espontaneidad y 
creatividad en la labor evangelizadora. Dentro de estas destacan gestas como la de Vasco de Quiroga en México o prédicas como la de Bartolomé de las Casa, quienes, en palabras de Klaiber, dejaron claro que "evangelizar no significaba solo anunciar la Palabra sino, sobre todo, construir una nueva sociedad basada en la caridad y la justicia” (p. 149). Luego, Klaiber señala que "Lamentablemente, esta fase utópica cedió a una etapa ideológica a mediados del siglo... el Concilio de Trento (1545-1563) dio inicio a una etapa caracterizada por una nueva ortodoxia que no toleraba críticas dentro de la Iglesia” (p. 149).

A partir de este punto, Klaiber resalta la labor de la Compañía de Jesús en el contexto tridentino. Resulta interesante contrastar la forma como abordó dicha labor en el volumen dedicado a la historia jesuita (Klaiber, 2007). Allí, luego de una sobria narración del protagonismo de determinados personajes como José de Acosta, Blas Valera o Juan Pablo Viscardo y Guzmán, Klaiber concluye que:

desde José de Acosta en el siglo XVI hasta Viscardo y Guzmán en el siglo XVIII, los jesuitas y sus simpatizantes buscaron la voluntad de Dios en la cultura y en la historia de las tierras donde se encontraban. Pero su comprensión de la historia y la cultura estuvo condicionada por su grado de simpatía hacia los pueblos que les tocaba servir. (p. 63)

Este lacónico juicio de la práctica jesuita contrasta con su descripción de la misma en el texto de la disertación, donde dice que, en el contexto tridentino

había cristianos que vivían durante esta transición de la etapa utópica a la nueva etapa ideológica; ellos no se dieron por vencidos y se dedicaron a preparar el camino para un nuevo período utópico. Un ejemplo fue la - por entonces - recién fundada orden, la Compañía de Jesús. (Klaiber, 2006, p. 149) 
Klaiber señala que la historia de los jesuitas durante la época colonial estuvo marcada por su obra en colegios y misiones como "un capítulo en la lucha entre utopía e ideología" (p. 156). Esta lucha se refleja, en primer lugar, en las instituciones educativas jesuíticas, en constante tensión con el poder virreinal, desde las pugnas con el virrey Toledo por el otorgamiento de títulos (p. 151). Klaiber resalta que durante la educación conservadora del Perú colonial, en el Colegio San Pablo "los jesuitas inculcaban a sus alumnos un sentido de orgullo acerca de sus capacidades y cualidades, dando origen así a una identidad criolla” (p. 156). De forma similar, en los colegios de caciques "se fomentaba la creación de una identidad indígena-cristiana... los hijos de caciques... se dedicaban al estudio de su propio idioma. También se les inculcaba orgullo de su pasado incaico" (p. 156).

Es claro que para Klaiber un elemento clave en la perspectiva utópica dentro de la enseñanza es forjar en los estudiantes una identidad que los convierta en personajes proactivos, con un actuar trascendente en sus comunidades que supere comportamientos conformistas. Esta enseñanza era, además, una invitación a la vida en libertad como condición para el desarrollo de las capacidades personales. No es sorpresa, por lo tanto, que una formación de estas características genere posteriores iniciativas independentistas. Como el mismo Klaiber señaló en cierto momento, "si San Antonio en el Cuzco preparaba a los guerrilleros de la independencia, San Carlos en Lima formaba a sus intelectuales" (1987, p. 168).

Klaiber ilustra otras dimensiones de la perspectiva utópica en la labor misional jesuita. Destaca la opción de la Compañía por evitar tener muchas doctrinas en el Virreinato y focalizarse en una cuantas que pudiesen convertirse en "modelos de la vida cristiana” (2006, p. 152). Klaiber ve aquí un deslinde entre dos formas de asumir la obra evangélica. En un caso, la administración de doctrinas llevaba al mantenimien- 
to de formas de vida y de evangelización preestablecidas, lo que contribuía al mantenimiento de un orden de cosas dado, en clara perspectiva ideológica. En el otro, se trataba de asumir la labor evangélica como una oportunidad de crear algo diferente que, como modelo, fuese una inspiración para otras doctrinas. No es difícil ver en esta lectura de Klaiber la convicción de que los jesuitas optaban por la perspectiva utópica.

Esta perspectiva es ilustrada con los casos de Juli y el Paraguay. En el primer caso, a partir de un breve resumen, Klaiber destaca el éxito económico de la misión de Juli, así como la implementación de una escuela para los jóvenes aymaras y la construcción de un hospital. La utopía implica, en este caso, la mejora concreta de las condiciones de vida de las personas y, una vez más, el acceso a la educación. El otro aspecto que resalta Klaiber es el de la inculturación, entendida como el proceso por el cual los evangelizadores incorporaban en su formación la cultura de los pueblos evangelizados. El reconocimiento y el diálogo con los otros aparece, en el texto, como un componente fundamental de la perspectiva utópica (pp. 152-153).

El bienestar económico, junto con el intercambio cultural, como fruto del reconocimiento del otro en la convivencia, son también características que Klaiber resalta de las misiones paraguayas. En este caso, sin embargo, se extiende con mayor detalle en las estrategias políticas y militares desplegadas por los jesuitas para la protección de las misiones, que incluía el entrenamiento de los guaraníes en tácticas bélicas modernas, así como en el uso de armas. Deseo resaltar este aspecto porque, al parecer, Klaiber otorga legitimidad al uso de la violencia en defensa de la obra utópica, frente a amenazas exteriores. Esta toma de posición refleja, a mi entender, que la perspectiva utópica que propone Klaiber no cae en la tentación idealista de una práctica aséptica, ajena a las contradicciones y riesgos de la sociedad humana en general. 
Klaiber nota este contexto al resumir su evaluación de las misiones jesuitas, indicando que "fueron enclaves utópicos en medio de un orden ideológico que no respetaba ni la dignidad ni los derechos de los indios. Los colegios para los criollos y los caciques fueron espacios privilegiados donde cada grupo podía forjar una nueva identidad como líderes cristianos (p. 157). Klaiber aborda en diversas partes de la disertación el accionar de la Compañía de Jesús que, por un lado, aparecía como soporte del régimen virreinal y, por otro lado, como su cuestionador. Al respecto, plantea que:

los jesuitas coloniales sabían que no podían desafiar al poder sin poner en peligro sus obras. Tenían que sujetarse al mundo real y crear proyectos creativos con prudencia dentro de un mundo que sospechaba de proyectos fuera de lo común. Pero el ideal siempre fue cambiar ese mundo y tomar los pasos necesarios para llegar a su utopía. (p. 157).

Más allá de la visión comprensiblemente positiva del accionar jesuita en la historia virreinal, Klaiber ilustra en este texto no solo su convicción a favor de la utopía como forma de asumir protagonismo en la historia, sino también cómo entiende la perspectiva utópica en la praxis personal.

En primer lugar, se trata de una acción que desafía un estado de cosas dominado por la perspectiva ideológica. Es un acto de rebeldía, guiado por la convicción de que la realidad puede ser diferente y que es posible generar esa diferencia. En segundo lugar, se trata de una acción creativa, abierta a innovaciones, a imaginar escenarios alternativos a los conocidos y a generar la forma de concretarlos. Es diferente del comportamiento rebelde focalizado en la negación de órdenes preestablecidos y su disolución, pero sin horizonte de escenarios alternativos. En tercer lugar, la construcción de una realidad utópica solo se gesta asumiendo una actitud de reconocimiento del valor del otro. Es un reconocimiento que va más allá de la tolerancia o la aceptación, porque está abier- 
to a descubrir y aprender nuevos conocimientos, enfoques, perspectivas y sensibilidades, a la vez que se comparten los propios.

Es importante recordar que la disertación parte proponiendo un paralelo entre el siglo XVI y el siglo XIX, contrastando el proceso de evangelización con el de la globalización. En tal sentido, la perspectiva utópica, con sus dimensiones de rebeldía, creatividad y apertura, se convierte en una invitación a una lectura del actuar en tiempos contemporáneos. Esto lo presenta Klaiber de forma explícita al concluir que "En este momento en que estamos... nos toca mantener viva la antorcha de la utopía: sabemos, como ha ocurrido anteriormente, que esta etapa ideológica ha sido superada por una etapa utópica” (p. 157).

Existen textos que informan y son buenos. Existen otros que informan e interpelan y son mejores que los primeros. Existen, por último, textos que informan, interpelan e inspiran. La lección inaugural de Jeffrey Klaiber en 2005 fue uno de esos, una invitación a vivir la utopía dentro del claustro universitario, como testimonio imperecedero de su dedicación docente y su compromiso como historiador.

Recibido: 03 de enero de 2020

Aprobado: 15 de marzo de 2020

\section{Referencias bibliográficas}

KLAIBER, J.

(1987) La Iglesia ante la emancipación en el Perú. En J. Klaiber S. J., (Ed.) Historia general de la Iglesia en América Latina (v. III, pp. 161-202). Salamanca: CEHILA. 
KLAIBER, J. (Ed.).

(1987) Historia general de la Iglesia en América Latina. Volumen III: Perú, Bolivia y Ecuador. Salamanca: CEHILA.

KLAIBER, J.

Iglesia, dictaduras y democracia en América Latina. Lima: Pontificia Universidad Católica del Perú.

KLAIBER, J.

(2006) Globalización y evangelización en los siglos XVI y XXI. Ideología frente a utopía. En V. Santuc (Comp.), La identidad ignaciana de la Universidad Antonio Ruiz de Montoya (pp. 147-157). Lima: Universidad Antonio Ruiz de Montoya.

KLAIBER, J.

(2007) Los jesuitas en América Latina, 1549-2000. Lima: Universidad Antonio Ruiz de Montoya.

KLAIBER, J.

(2013) El clero ilustrado en tiempo de Abascal. En S. O’Phelan y G. Lomné (Eds.), Abascal y la contraindependencia de América del Sur (pp. 75-87). Lima: Instituto Francés de Estudios Andinos, Pontificia Universidad Católica del Perú.

296 MANHEIM, K.

(1973) Ideología y utopía. Introducción a la sociología del conocimiento. Madrid: Aguilar. 